\title{
Vivre dans le silence : Histoires inédites de violences sexuelles
}

\section{par Lily Crist}

Leçons tirées de femmes réfugiées victimes de violences sexuelles liées au conflit au Kosovo et application de la science médico-légale aux soins dispensés aux populations de Vancouver.

$\mathrm{E}$ n grandissant, j'entendais des histoires que des adultes racontaient, des histoires qui me tenaient éveillé la nuit. Ils m’ont intrigué par la complexité des mots qui m'étaient inconnus et m'ont enveloppé d'émerveillement et de crainte. Si vous arriviez à vous échapper de ce monde d'adultes, ou si vous prétendiez être en train de jouer et ne pas faire attention au monde qui vous entoure pendant qu'ils partagent leurs histoires, alors vous seriez en mesure d'en apprendre davantage sur leur monde. Toute personne qui dit que les enfants ne comprennent pas ce qui se passer dans le monde des adultes se trompe.

Comme dans la Grèce antique et dans de nombreuses cultures méditerranéennes, le gynécée était un domaine fermé pour les femmes où elles pouvaient être libres de se réunir et de partager des histoires. Le gynécée était souvent une pièce ou un lieu dans une maison où les femmes, mariées et célibataires, esclaves et libres, pouvaient se réunir et passer du temps entre elles. Personnellement, le gynécée, et tout autre rassemblement de femmes, a permis d'apporter au monde de nouveaux mots et de nouvelles idées. Au fil du temps, une géographie particulière a commencé à émerger lorsque j’ai appris comment les hommes et les femmes interagissaient et comment les attentes liées au genre fondées sur les traditions, les coutumes et la moralité avaient un impact sur moi et les personnes de mon entourage. Durant mon enfance, j'ai appris que certaines histoires devaient rester inexpliquées de peur de représailles ou pire; elles étaient si toxiques qu'elles pouvaient avoir des impacts désastreux. Parfois, ces histoires apparaissaient par fragments, de façon incomplète et mystérieuse, puis se propageaient dans tous les domaines de la vie d'une personne. Que se passe-t-il quand la vérité est si insupportable à raconter et, pour continuer à vivre, il faut enterrer son histoire au plus profond de soi? Que se passe-t-il quand il n'y a personne à qui parler?

$\mathrm{Au}$ fil des décennies, il me paraissait très important de comprendre les obstacles auxquels une victime est confrontée lorsqu'elle choisit de raconter son histoire - ou lorsqu'elle est incapable de le faire - et les meilleurs moyens de prendre soin des victimes de violence sexuelle. Ce document porte principalement sur les femmes réfugiées du Kosovo et sur les soins qui leur sont fournis. En examinant leur situation, j'espère en apprendre davantage sur les difficultés d'accès aux soins et sur les moyens d'améliorer, pour cette population spécifique, l'accès à des soins adéquats.

Ce document essaye de mettre en lumière certaines approches et réponses relatives aux soins de ces femmes réfugiées victimes de violences sexuelles dans les situations de conflit et au rôle des sciences médico-légales. L'accent sera mis sur un groupe spécifique : les femmes d'origine albanaise de la région du Kosovo avant et durant les guerres dans les Balkans, principalement des réfugiées victimes de violences sexuelles. La violence sexuelle peut en effet être liée aux conflits, comme lors des guerres balkaniques. De nombreux parallèles peuvent être établis avec d'autres femmes dans d'autres zones de guerre où les forces militaires ont utilisé la violence sexuelle comme outil contre les populations civiles. Selon la Résolution 1820, S/RES/1820 (2008) du Conseil de sécurité des Nations unies, depuis le 19 juin 2008, la violence sexuelle est liée à des conflits armés « quand elle est utilisée ou commanditée comme arme de guerre prenant délibérément pour cible des civils, ou dans le cadre d'une attaque généralisée ou systématique dirigée contre des populations civiles. » La résolution indique que les « civile constituent la grande majorité de ceux qui subissent les effets préjudiciables des conflits armés; (...) les femmes et les filles sont particulièrement ciblées par l'utilisation de violences sexuelles, y compris comme tactique de guerre destinée à humilier, dominer instaurer de la peur, disperser ou déplacer contre leur gré les membres civils d'une communauté ou d'un groupe ethnique. »

Avec ce format limité, il est assez difficile et complexe d'expliquer comment une société spécifique fonctionne et comment cela a trait aux femmes victimes de violences sexuelles. Néanmoins, nous pouvons examiner certaines des raisons pour lesquelles les victimes de viol, dans ce contexte spécifique, ne divulguent pas leur situation aux prestataires de soins de santé ou aux agents de la force publique. Nous examinerons ensuite des stratégies pour répondre aux besoins des femmes réfugiées. En se concentrant sur les soins dont les femmes victimes ont besoin, en ce qui a trait au conflit dans les Balkans, il est possible de mieux comprendre les chemins que les prestataires de soins de santé (infirmiers et infirmières médico-légaux) ont pris ou peuvent prendre. Au fil des années, le Canada a accueilli de nombreux réfugiés de zones de guerre, et une meilleure compréhension des femmes réfugiées et de leurs besoins peut avoir un impact positif sur les prestataires de soins de santé et leurs patients. Par le passé, le Canada a été directement impliqué sur le terrain au Kosovo en fournissant des équipes militaires, médicales et civiles.

Pour mieux aider à comprendre certains des obstacles relatifs à l'accès aux soins, une courte introduction anthropologique pour établir le contexte du conflit et une brève description de la société albanaise et des opinions sur les femmes victimes sont nécessaires. Premièrement, voici quelques informations de base pour mieux comprendre la culture et les traditions albanaises au Kosovo.

Dès que Slobodan Milošević est arrivé au pouvoir dans l'ex-Yougoslavie dans les années 1990, avant même le début de la guerre dans les Balkans, la population d'origine albanaise de 
la région du Kosovo vivait sous la contrainte. Les prestataires de soins de santé, les enseignants et de nombreux Albanais de la région ont perdu leur emploi simplement parce qu'ils étaient albanais. Les écoles, les universités, les hôpitaux et toutes les organismes gouvernementaux dirigés par des Albanais de souche pour leurs populations ont été fermés, et ce pour les dix années suivantes, jusqu'à la fin du conflit dans les Balkans. Dans les hôpitaux locaux, Les prestataires de soins de santé d'origine albanaise ont été remplacés par des Serbes, des gens que la population albanaise locale se méfiait grandement. À cause de ce contexte, une société illégale parallèle a tenté de résister à l'occupation. Les soins prénataux étaient quasi inexistants et pour beaucoup de femmes albanaises accoucher devenait une source de peur et de danger. Avec une telle situation sur le terrain, les femmes ont décidé de ne plus partager leurs problèmes avec des prestataires de soins de santé en qui elles n'avaient aucune confiance.

La violence à l'encontre des populations civiles a augmenté au Kosovo et a atteint son point culminant durant la guerre de 1998-1999. Dans l'article « Combien de temps pouvons-nous garder un secret? En ce qui a trait aux victimes de viol durant la guerre au Kosovo, la réponse est probablement jamais »de Radio Libre Europe / Radio Liberté. Les viols commis par les forces paramilitaires Serbes avaient pour objectif, en partie, de terroriser, d'humilier et de stigmatiser les femmes (Rames, 2013). La guerre a finalement pris fin, mais les difficultés ont continué pour les survivants. Les attitudes à l'égard des victimes de violences sexuelles sont très négatives. Il est clair que la société albanaise a certainement maintenu certaines croyances flagrantes. L'une d'elles est que l'honneur d'une femme albanaise est plus précieux que sa vie et qu'une « bonne » femme doit se suicider après une agression sexuelle pour éviter de faire honte à sa famille et à son clan. Avec une telle mentalité, la stigmatisation a un impact sur la capacité - et la possibilité - des victimes de se faire soigner.

La société albanaise de la région du Kosovo était une société à dominance masculine rigide et codifiée, dans laquelle les femmes passaient de l'autorité de leurs pères, frères et autres parents de sexe masculin à celle des familles de leur mari ou de leur belle-famille. La prévalence de l'ancien code des lois traditionnelles, le Kanun du XV siècle, qui régissait les questions relatives à la famille, au mariage, aux biens, à la vie de clan, etc., avait une incidence sur les relations entre les sexes et les opinions sur les victimes de violences sexuelles et domestiques. Durant leur fuite, de nombreuses familles albanaises du Kosovo ont été persécutées et déplacées dans les pays voisins ou ont été tuées pendant la guerre. Parmi les femmes réfugiées albanaises, les récits de violence et de violence sexuelle n'ont pas été partagés avec le monde extérieur ni même avec les époux ou d'autres membres de la famille, par peur d'être abandonnées, soumises à des représailles ou d'être rejetées par leurs communautés. La violence sexuelle était et est toujours un sujet tabou dans cette société. Depuis août 2013, le Centre de réhabilitation des victimes de torture du Kosovo (KRCT) collabore à un projet intitulé « Renforcer le pouvoir des femmes victimes de violences sexuelles et de tortures pendant le conflit au Kosovo » aider les victimes à se faire connaître et à recevoir une indemnisation et des conseils médicaux.
Dans l'étude commandée par les Nations Unies et intitulée Healing the Spirit: Reparations for Survivors of Sexual Violence Related to the Armed Conflict in Kosovo (La guérison de l'esprit : Réparations pour les survivantes de violences sexuelles liées au conflit armé au Kosovo), la peur de la stigmatisation et les conséquences sociales qui en découlent sont des raisons spécifiques pour lesquelles les victimes n'ont pas révélé d'incidents de violence sexuelle. En effet, en se présentant, elles seraient ostracisées par toute la famille et leur communauté; leurs conjoints et leurs familles les abandonneraient et les considéreraient comme des parias. En outre, la honte ressentie par les victimes de violences sexuelles est l'un des raisons pour lesquelles elles ne partagent pas leurs histoires. Selon l'article intitulé « Tracking Gender-Based Human Rights Violations in Postwar Kosovo » du American Journal of Public Health, la crainte d'être stigmatisé a été aggravée - et l'est encore à ce jour - par le manque de services de soutien et comme principal obstacle à la divulgation des violences sexuelles (Sapna et Perry, 2004). Outre les obstacles habituels liés à l'accès aux soins, les victimes de ce conflit ont été confrontées à une stigmatisation accrue de la part de leurs communautés et de leurs familles. « La guérison de l'esprit » mentionne également le manque de prestataires de soins de santé après le conflit en raison de leur exécution pendant la guerre et de la destruction générale des infrastructures.

Ce conflit a permis de dégager de nouveaux moyens importants pour prendre en charge les femmes réfugiées victimes de violences sexuelles. L'une des approches mentionnées dans le « Guttmacher Report in Public Policy » de 1999 était la distribution de trousse de santé reproductive aux réfugiées kosovares au moment où commençaient à apparaître des cas de viols systématiques de femmes albanaises quand elles essaient de quitter leur pays (The Guttmacher Report on Public Policy, 1999).

Dans son article de 2014 publié par Nursing intitulé « Female Refugees: Sensitive Care Needed » (Les femmes réfugiées: des soins sensibles requis), Elizabeth Heavey fournit une meilleure compréhension des défis posés par les soins infirmiers et des stratégies utiles pour prendre soin des femmes réfugiées. Les patients qui ont subi des violences sexuelles pendant les guerres peuvent montrer des signes de trouble de stress post-traumatique (TSPT) et utiliser la dissociation « comme un mécanisme d'adaptation $\gg$. Dans l'article, Heavey décrit certains des facteurs de risque pour la santé observables chez les victimes souffrant de TSPT. Ils vont des troubles cardiovasculaires au suicide. Comme elle le note, « le TSPT n’affecte pas seulement la santé mentale ». Selon Linda E. Ledray (2011) dans « Medical Response to Agression Sexual Adult », les réactions à des événements traumatisants, tels que la violence sexuelle, peuvent prendre plusieurs formes : physique, émotionnelle, cognitive et comportementale (pp. 215-216).

Heavey explique que les infirmiers et infirmières peuvent jouer un rôle clé dans l'aide aux victimes. Selon elle, « une communication claire et une empreinte de compassion sont essentielles pour optimiser les soins infirmiers et médicaux ». Quand les patients connaissent les prestataires de soins de santé et se sentent en sécurité avec eux, les victimes ont plus tendance à 
vouloir partager leurs histories. Tel est ce que Bridgid McGowan a déclaré lors d'une conférence donnée à l'Institut de technologie de la Colombie-Britannique le 17 février 2015 dans le contexte d'un cours intitulé «Sexual Assault Nurse Examiner Core Education: Theoretical Aspects $\gg$ (Enseignement de base des infirmières examinatrices en matière d'agression sexuelle Aspects théoriques).

Dans sa présentation, McGowan a expliqué comment les infirmiers et infirmières en médecine légale et autres infirmiers et infirmières peuvent aider les victimes de traumatismes en leur donnant le contrôle du processus médical, créant ainsi un sentiment de prévisibilité et en prévenant la perte de sécurité. Ce cours a été enseigné par Aimee Falkenberg (RN FNE BSN SANE-A). Lors d'une discussion avec moi, elle a partagé une partie de la sagesse et de l'expérience qu'elle avait acquises grâce à son travail auprès des victimes d'agression sexuelle et de traite de personnes. Elle a soulevé des points importants concernant l'aide aux victimes. Elle nous a notamment conseillé de ne pas demander à une survivante de répéter son histoire à un trop grand nombre de personnes, car il est déjà assez difficile de ne la raconter une fois. Falkenberg met l'accent sur le renforcement de la victime en lui indiquant à quel point elle peut être fière d'être venue chercher de l'aide. Elle a également évoqué les nombreux obstacles rencontrés par les victimes qui tentent d'accéder à des soins à la suite de violences sexuelles. Très peu de victimes viennent chercher de l'aide, il est donc primordial de soutenir celles qui le font. Elle a ajouté qu'en tant qu'infirmières et personnel médical, nous devons être attentifs et éviter de juger ou de décider si des violences sexuelles ont eu lieu ou non. Nous sommes sur place pour fournir à la victime des besoins médicaux correspondant à ses antécédents et pour lui offrir une voie vers des services de soins infirmiers médico-légaux, permettant ainsi à la victime de garder le contrôle à tout moment. Falkenberg a également partagé un élément clé de toute interaction avec les victimes, la nécessité d'écouter. Lorsque nous écoutons, nous montrons de la compassion et une victime est plus susceptible de s'ouvrir, en partageant une anecdote ou une histoire pertinente.

La criminalistique ne fonctionne pas seulement en soins infirmiers. Cela peut également s'appliquer à des interventions sociales comme celles qui se déroulent à La Boussole, une organisation francophone à but non lucratif de Vancouver qui, entre autres, aide les femmes réfugiées à s'intégrer dans la société canadienne. Pendant un an, j’ai été directrice exécutive de La Boussole. Chaque semaine, les francophones nouvellement arrivés dans la ville nous contactaient. Ils avaient besoin d'aide pour obtenir un logement et un travail, des conseils, des conseils financiers et des services d'interprétation. Nos clients se heurtaient à de nombreux obstacles pour accéder aux soins de santé et autres services. En effet, les professionnels de la santé de la ColombieBritannique ne comprenaient pas forcément les langues parlées par les réfugiés que nous rencontrions régulièrement. Par conséquent, nos client pouvaient avoir du mal à se faire comprendre et plus généralement à recevoir les soins physiques et mentaux dont ils ont besoin.

$\mathrm{Au}$ sein de cet organisme, les employés accompagnent leurs clients vers diverses institutions, y compris les cabinets de médecins et les hôpitaux, jouant le rôle d'interprètes et de guides dans leurs interactions avec le personnel médical. Une telle aide est importante car les réfugiés à Vancouver, en particulier les femmes, se heurtent à des obstacles uniques. Par exemple, il peut ne pas y avoir d'espace dans les maisons de transition ou même pas de maisons de transition dans leurs quartiers. Elle peuvent ne pas savoir comment contacter ces lieux ou être capables d'interpréter les règles une fois qu'elles ont été admises. En outre, leurs expériences dans leurs pays d'origine pourraient les amener à se méfier des forces de l'ordre et du système judiciaire.

À La Boussole, les employés possèdent les connaissances et l'expérience requises pour aider les réfugiés arrivant dans la ville. Ils peuvent écouter les réfugiés de manière impartiale et agir conformément aux protocoles bien établis en matière de travail social. Chaque employé apporte sa touche personnelle et ses expériences de vie lorsqu'il aide des réfugiés en les guidant dans le dédale bureaucratique, les empêchant parfois de recevoir des soins de santé adéquats. Nos employés peuvent adapter leurs interventions à chaque client sur la base de leur empathie envers ceux qu'ils aident, de leur connaissance de la communauté et de leurs différents repères socioculturels. Leur capacité à adapter leurs approches en fonction des besoins des différents clients est essentielle, car ces besoins peuvent varier considérablement. Les caractéristiques personnelles des clients sont un facteur, de même que la gravité de leurs expériences récentes, immersives et graves, de la guerre et des conflits. Récemment, à La Boussole, nous avons aidé des femmes, souvent accompagnées de leurs enfants, qui avaient fui des situations de violence dans leur pays d'origine, où les agressions sexuelles étaient une pratique très courante. Certaines clientes ont également fui la violence vécue au Canada.

Le personnel de La Boussole accompagne les clients pour leurs rendez-vous médicaux. Dans ce contexte, les membres du personnel ne font pas simplement office d'interprètes pour le compte des clients. Par leur soutien et leurs conseils moraux, ils aident à rendre les interactions du client avec le personnel médical aussi harmonieuses que possible. Tout en respectant l'autonomie de leurs clients, ils s'efforcent de faire en sorte que leurs points de vue soient exprimés et que leurs préférences soient défendues. Ils aident également les clients à donner suite aux conseils, aux ordonnances et aux visites de suivi avec un médecin ou des spécialistes.

Ma propre expérience éducative et professionnelle ma bien préparée à accompagner et guider les clients et faire face aux défis et même aux crises qui se produisent de temps à autre. En particulier, mes études en sciences de la santé à l'Institut de technologie de la Colombie-Britannique mont permis d'acquérir les connaissances et la confiance nécessaires pour soutenir, du mieux que je peux, les femmes qui se sont échappées des zones de conflit et de violence interpersonnelle. Lorsque quelqu'un appelait ou se présentait à La Boussole et se sentait à l'aise de partager ses expériences avec moi, il était important pour moi d'écouter attentivement, de faire preuve d'empathie et d'observer les détails révélateurs partagés par le visiteur. En faisant cela, j'étais en mesure d'établir un plan d'action adapté à ses besoins. En effet, en prêtant une attention particulière à l'histoire de sa vie et en écoutant ce qu'elle soulignait, je pouvais rapidement élaborer un plan adapté à ses besoins. 
Les sciences médico-légales en matière de soins infirmiers ont évolué au cours des dernières années pour mieux clarifier les problèmes épidémiologiques, tels que les crimes violents (de l'agression sexuelle au meurtre), les traumatismes, la négligence et le suicide; pour n'en nommer que quelques-uns. La criminalistique liée aux soins infirmiers est très pertinente pour les personnes que l'on rencontre si fréquemment dans notre société, dans les cliniques d'accueil, les salles d'urgence et ailleurs.

Plus généralement, l'intervention revêt de nombreuses facettes car elle implique une diversité d'experts travaillant dans des domaines interdépendants. Les personnes qui aident les victimes travaillent entre autres dans les domaines de l'application de la loi, de la justice, de la médecine et du travail social. Une formation en sciences médico-légales peut aider les gens dans tous ces domaines. Cela ma certainement aidé.

La criminalistique contribue à une meilleure compréhension des signes de violence - qui peuvent différer en fonction des caractéristiques d'une victime, notamment de son âge - et à une meilleure capacité de poser les bonnes questions. Pour réussir à aider les femmes victimes de maltraitance physique ou mentale, il faut être curieux et ouvert d'esprit. Il faut également se documenter et préserver les preuves de préjudice. La familiarité avec la criminalistique peut permettre à un professionnel de la santé d'aider plus efficacement les victimes d'agression sexuelle, de crimes violents ou d'autres formes de maltraitance. À cet égard, certains hôpitaux du Lower Mainland proposent des services de soins adaptés aux besoins des victimes d'abus sexuels (infirmière examinatrice en matière d'agression sexuelle [IEAS]).

$\mathrm{Au}$ BCIT, la professeure Sheila Early m'a appris qu'un esprit curieux, une écoute active et un devoir de sollicitude sont au cœur des soins infirmiers légistes et que nous avons tous un rôle à jouer pour mettre fin à la violence dans la société, que ce soit une clinique ou dans des conversations entre amis. Dans ses enseignements, la professeure Early a souvent insisté sur le fait qu'un infirmier examinateur légiste devait être perspicace et interroger constamment les circonstances dans le but de découvrir la vérité afin de soutenir les victimes le plus efficacement possible. Nos clients ne disent pas toujours ce qui les trouble, mais un auditeur instruit et expérimenté peut souvent aller au cœur de leurs problèmes. À cet égard, les femmes réfugiées ayant survécu à une agression sexuelle et à d'autres formes de violence pourraient consulter un médecin pour des problèmes sans lien avec leurs expériences traumatiques tout en présentant des symptômes de trouble de stress post-traumatique, notamment d'hypervigilance. Un professionnel perspicace peut détecter ces symptômes et adapter son traitement en fonction de son interprétation de la situation.

Malheureusement, les expériences des réfugiés albanais du Kosovo qui se sont installés au Canada ne sont pas exceptionnelles. De nombreuses femmes réfugiées d'autres zones de difficultés et de conflits dans le monde, y compris des camps de réfugiés, sont confrontées aux mêmes problèmes et présentent des risques qui leur sont propres. De plus, on peut appliquer ses connaissances en sciences médico-légales à des personnes qui fuient des situations difficiles au Canada, qu'elles vivent dans des communautés éloignées ou dans les quartiers urbains et suburbains défavorisés des grandes villes. Cependant, le nombre de femmes réfugiées qui sont arrivées au Canada en provenance de l'extérieur du pays reste élevé, avec 28689 en 2016 seulement (gouvernement du Canada, 2017). Chaque réfugiée apporte avec elle des traumatismes spécifiques et fait face à des défis particuliers.

Le travail des infirmières examinatrices judiciaires et les approches sensibles à la culture, parmi toutes les personnes qui aident les réfugiés, sont deux moyens d'aider au processus de guérison. Pour les femmes survivantes du Kosovo, l'accès à la justice et les programmes de réparation figurent parmi les outils élaborés par le Secrétaire général des Nations Unies en 2014 dans le cadre de sa Note d'orientation sur les « réparations des violences sexuelles liées aux conflits ». Siobhan Hobbs, Conseillère pour les questions de genre au Bureau des projets des Nations Unies pour les femmes au Kosovo, a déclaré: « Il s'agissait en réalité d'une tentative du chef des Nations Unies de mettre en commun les leçons tirées, non seulement en ce qui a trait aux violences sexuelles, mais aux réparations en général $\gg$.

La dévastation d'une guerre se poursuit longtemps après sa fin et de nombreuses initiatives et programmes gouvernementaux sont mis en place pour aider les victimes des conflits, reconstruire leurs infrastructures et créer une société plus juste, caractérisée par l'absence d'inégalités ou de violences fondées sur le sexe. Cependant, dans un rapport du HCR, la situation des femmes albanaises après la guerre est perçue comme s'être détériorée et, en outre, certaines jeunes femmes appartenant à des groupes socio-économiques défavorisés sont devenues victimes de la traite d'êtres humains (Baker \& Hilde, 2002).

Certaines histoires nont peut-être pas encore été racontées ou peuvent être ensevelies sous un lourd mur de silence. Depuis mon enfance, j'ai continué à écouter de telles histoires, maintenant plus pour aider les victimes à trouver le courage nécessaire pour exprimer ce qu'elles ressentent au plus profond d'ellesmêmes. Dans beaucoup de cas, ce sont leurs silences qui sont les plus éloquents. Pour elles, revisiter les maux que la guerre et les conflits à créer peuvent entraîner l'ouverture la boîte de Pandore des temps modernes. Cependant, les femmes réfugiées du Kosovo victimes de violences sexuelles doivent être en mesure de trouver l'aide dont elles ont tellement besoin pour avoir l'espoir de reconstruire une meilleure vie. En albanais, ils l'appellent cela «Shpresē » et les petites filles sont parfois appelées «Shpresa », celle qui a de l'espoir.

\section{Remarques de l'auteur}

1. Une communication claire et une empreinte de compassion sont essentielles pour optimiser les soins infirmiers et médicaux.

2. Les infirmières peuvent aider les victimes de traumatismes en leur donnant le contrôle du processus médical, créant ainsi un sentiment de prévisibilité et en prévenant la perte de sécurité.

3. Renforcer le pouvoir des survivantes en leur faisant savoir à quel point elles peuvent être fières d'être venues chercher de l'aide

4. Nous devons être attentifs et éviter de juger ou de décider s'il y a eu ou non violence sexuelle.

5. L'écoute est un élément essentiel d'interaction avec les victimes. 


\section{Au sujet d'auteure}

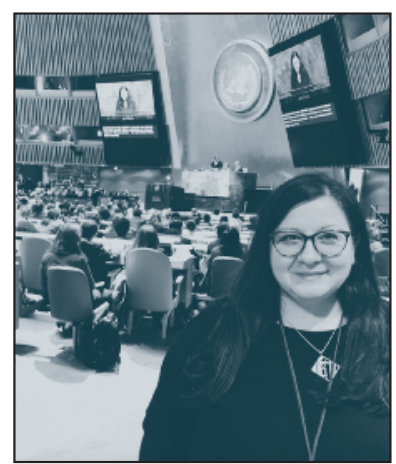

À titre de présidente de l'Alliance des femmes de la francophonie canadienne, Lily Crist travaille fort pour représenter les femmes francophones au Canada dans les neuf provinces et les trois territoires, où elles font partie d'une minorité linguistique. Elle est impliquée dans le mouvement des femmes depuis 22 ans. Mme Crist a étudié en tant qu'éducatrice pour adultes, conseillère et en sciences médico-légales, ce qui la conduit à être une partisane expérimentée et bien informée des victimes de violences sexuelles. Mme Crist a aidé à élaborer un projet pilote d'éducation qui apporte de l'information sur les relations saines, le genre et la diversité aux écoles secondaires francophones. En travaillant avec La Boussole, Mme Crist fait partie du seul organisme francophone à lutter contre l'itinérance et à offrir des services aux personnes vivant dans la pauvreté par le biais d'une banque alimentaire, de cours communautaires, d'appuis à des initiatives en santé mentale et par la création d'espaces francophones sécuritaires et ouverts à Vancouver. En outre, Mme Crist a contribué à la mise en place d'une ligne d'assistance francophone pour les crises, Inform'elles, qui offre un soutien aux femmes francophones vivant dans les régions anglophones. Depuis septembre 2018, elle poursuit des études en sciences infirmières au V.C.C. à Vancouver.

\section{RÉFÉRENCES}

Baker, J., \& Hilde H. (2002, November). The Kosovo Women's Initiative: An Independent Evaluation. Geneva, Switzerland: Evaluation and Policy Analysis Unit, United Nations. Retrieved from: http://www.unhcr.org/cgibin/texis/vtx/home/opendocPDF. pdf? docid=3db019784\&query=Kosovo

Desai, S., \& Perry M.J. (2004). Tracking Gender-Based Human Rights Violations in Postwar Kosovo. American Journal of Public Health, 94(8), 1304-1307. Retrieved from: http://www.ncbi.nlm.nih. gov/pmc/articles/PMC1448442/

Falkenberg, A. (2018, August 13). Follow up on Sexual Assault Nurse Examiner Core Education: Theoretical Aspects. Burnaby, BC: BCIT.

Government of Canada. (2017, November 1). 2017 Annual Report to Parliament on Immigration [not available]. Retrieved from https://www.canada.ca/en/immigration-refugees-citizenship/ corporate/publications-manuals/annual-report-parliamentimmigration-2017.html

Heavey, E. (2014). Female Refugees: Sensitive Care Needed. Nursing, 44(5), 28-34.

Hobbs, S. (2016, August). Path to justice for survivors of Kosovo's sexual violence: Q\&A with Siobhan Hobbs. Retrieved from: http://eca.unwomen.org/en/news/ stories/2016/08/q-and-a-with-siobhan-hobbs
Ledray, L.E., Burgess, A.W., \& Giardino, A.P. (2011). Medical Response to Adult Sexual Assault: A Resource for Clinicians and Related Professionals. St. Louis, MO: STM Learning, Inc.

McGowan, B. (2015, February 17). Sexual Assault Nurse Examiner Core Education: Theoretical Aspects. Burnaby, BC: BCIT.

Rames, V.S. (2013). Healing the Spirit: Reparations for Survivors of Sexual Violence Related to the Armed Conflict in Kosovo. Office of the United Nations High Commissioner for Human Rights. Retrieved from: http://www.ohchr.org/Documents/Issues/ Women/WRGS/PeaceAndSecurity/StudyHealingTheSpirit.pdf

The Guttmacher Report on Public Policy. (1999, June). Reproductive Health Kits Provided by UNFPA to Kosovar Refugees. The Guttmacher Report on Public Policy, 2(3), 12-13.

United Nations Security Council. (2008, June 19). Resolution 1820. Retrieved from: http://www.securitycouncilreport.org/atf/ cf/\%7B65BFCF9B-6D27-4E9C-8CD3-CF6E4FF96FF9\%7D/ CAC\%20S\%20RES\%201820.pdf 